\section{Commentary: Adding fuel to the fire for mechanical support during lung transplantation-More might be better}

Sara Najmeh, MD, and

Matthew G. Hartwig, MD, MHS

In their latest article in this issue of the Journal, Hoetzenecker and colleagues ${ }^{1}$ of the Vienna Lung Transplant group address a timely and controversial topic in the field of lung transplantation. They suggest that a single intraoperative technical consideration can significantly reduce primary graft dysfunction (PGD) in the newly implanted lungs.

The use of mechanical support during lung transplantation has been a subject of debate since the initial description of the procedure. Liberal use of cardiopulmonary bypass (CPB) during lung transplantation in the early years allowed reliable hemodynamic stabilization during the lengthy and complex procedure, improved visualization through cardiac decompression, and controlled early graft reperfusion. The use of CPB came with a price, however, that of increased coagulopathy, need for transfusion, reoperation for bleeding, and systemic inflammatory reaction, which itself was thought to contribute indirectly to early PGD. ${ }^{2}$ Given these increased risks, centers started shifting their practice toward a more selective use of $\mathrm{CPB}$, but identification of reliable preoperative predictors of need for intraoperative CPB support proved to be challenging.

As mechanical circulatory support technologies improved, venoarterial extracorporeal membrane oxygenation (ECMO) emerged as a potential hemodynamic support alternative to CPB during lung transplantation because it allowed oxygenation, ventilation, and circulation support without requiring the full systemic anticoagulation

\footnotetext{
From the Division of Cardiovascular and Thoracic Surgery, Department of Surgery, Duke University Medical Center, Durham, NC.

Disclosures: Authors have nothing to disclose with regard to commercial support.

Received for publication Jan 9, 2020; revisions received Jan 9, 2020; accepted for publication Jan 9, 2020; available ahead of print Jan 31, 2020

Address for reprints: Matthew G. Hartwig, MD, MHS, Duke University Medical Center 3863, Durham, NC 27710 (E-mail: Matthew.hartwig@duke.edu).

J Thorac Cardiovasc Surg 2020;160:328-9 0022-5223/ $\$ 36.00$

Copyright $(9) 2020$ Published by Elsevier Inc. on behalf of The American Association for Thoracic Surgery

https://doi.org/10.1016/j.jtcvs.2020.01.031
}

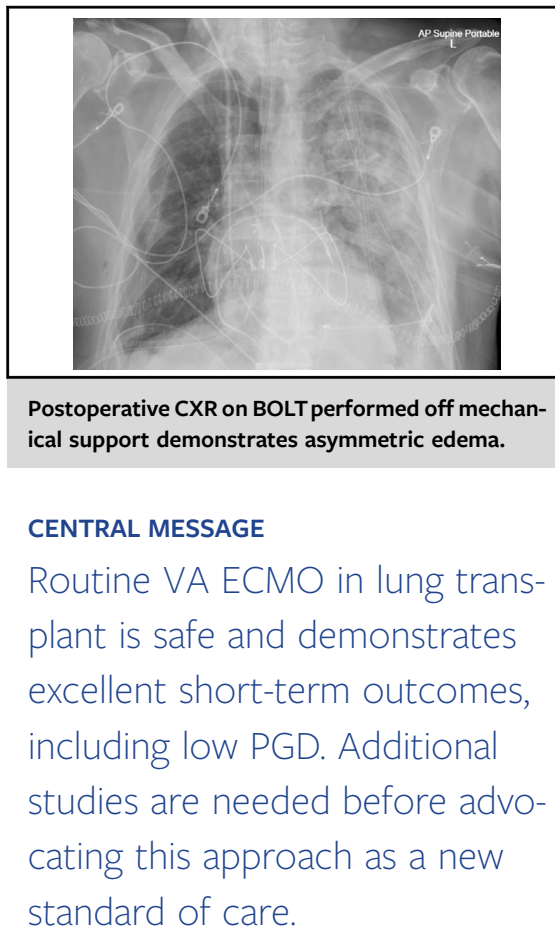

necessary for $\mathrm{CPB}$, thereby decreasing the risk of bleeding complications. ${ }^{4,5}$

As venoarterial ECMO became more widely used, its benefits appeared to extend beyond just reducing bleeding complications. Several series reported improved early graft function as well as long-term survival in patients who underwent lung transplantation with venoarterial ECMO support relative to those without ECMO. One of the largest series examining this subject was published by Hoetzenecker and colleagues ${ }^{6}$ in 2018, in which they reviewed their experience with 582 patients during a 6-year period ending in 2016 and demonstrated increased 1-, 3-. and 5-year survivals when venoarterial ECMO was used intraoperatively, as well as a significant trend toward reduced PGD in those patients.

Their experience led them to the question of whether venoarterial ECMO should be routinely used in all lung transplantation cases, a question that Hoetzenecker and colleagues ${ }^{1}$ have tried to address in this publication. The group adopted the "prophylactic" venoarterial ECMO approach in all their lung transplant recipients after 2016 and report here their prospectively collected data of the 159 patients who underwent transplant in the course of 2 years, focusing on PGD as the main outcome. Their results demonstrate remarkably low PGD rates, with the vast majority of patients being extubated or having a PGD of 0 in the first 72 hours. These benefits translated into an excellent 
2-year survival rate of $86 \%$. Although the short-term results presented in this article are intriguing, another key contribution of this work is the detailed and well-thought protocol for initiation and maintenance of intraoperative venoarterial ECMO. This includes the maintenance of ECMO intraoperatively, as well as the evidence of safety of the reduced-dose heparin protocol used by the group. Hoetzenecker and colleagues ${ }^{1}$ use only a single dose of heparin (40 units $/ \mathrm{kg}$ ) administered before central cannulation, with no thromboembolic events recorded in the entire series, thereby underlining the safety of this low-dose heparin protocol.

Although the data presented are suggestive that venoarterial ECMO may improve early perioperative outcomes in a select group of recipients, the conclusion that venoarterial ECMO should be used prophylactically in every lung transplantation operation cannot be made. Hoetzenecker and colleagues ${ }^{1}$ comment that the use of a control group of patients undergoing transplant without ECMO was not feasible because of lack of equipoise; however, in the end this works against their case, as it severely diminishes the power of these data. Furthermore, there is actually tremendous equipoise in the transplant community, as can be noted by the varied rates of routine versus selective mechanical support amongst centers. There are other concerns with generalizing the data presented here. For example, obstructive disease was the indication for transplant for most of the recipients in this study. This is a cohort of patients who usually has excellent short-term outcomes and low PGD rates regardless of intraoperative support. Other questions around the donors included in the study, such as the use of lungs from donation after circulatory death donors was only $5.6 \%$, make generalization of these data to other lung transplantation centers quite challenging. Finally, rates of bleeding and infectious complications in this series are still close to $10 \%$, a risk that is not insignificant in this tenuous population and one that should be taken into consideration.

In the end, the Vienna Lung Transplant Group has added fuel to the fire of regular venoarterial ECMO support during routine lung transplantation. Unfortunately, limitations of the study prevent us from advocating for this as a standard of care. Regardless of the generalizability of these results, however, the safety of the presented intraoperative venoarterial ECMO protocol and the importance of slow, controlled organ reperfusion after transplant are elegantly reiterated in this work.

\section{References}

1. Hoetzenecker K, Benazzo B, Stork T, Sinn K, Schwarz S, Schweiger T, et al; Vienna Lung Transplant Group. Bilateral lung transplantation on intraoperative extracorporeal membrane oxygenator: an observational study. J Thorac Cardiovasc Surg. 2020;160:320-7.e1.

2. Biscotti M, Yang J, Sonett J, Bacchetta M. Comparison of extracorporeal membrane oxygenation versus cardiopulmonary bypass for lung transplantation. $J$ Thorac Cardiovasc Surg. 2014;148:2410-5.

3. de Hoyos A, Demajo W, Snell G, Miller J, Winton T, Maurer JR, et al. Preoperative prediction for the use of cardiopulmonary bypass in lung transplantation. J Thorac Cardiovasc Surg. 1993;106:787-95; discussion 795-6.

4. Ius F, Kuehn C, Tudorache I, Sommer W, Avsar M, Boethig D, et al. Lung transplantation on cardiopulmonary support: venoarterial extracorporeal membrane oxygenation outperformed cardiopulmonary bypass. J Thorac Cardiovasc Surg. 2012;144:1510-6.

5. Machuca TN, Collaud S, Mercier O, Cheung M, Cunningham V, Kim SJ, et al Outcomes of intraoperative extracorporeal membrane oxygenation versus cardiopulmonary bypass for lung transplantation. J Thorac Cardiovasc Surg. 2015;149; 1152-7.

6. Hoetzenecker K, Schwarz S, Muckenhuber M, Benazzo A, Frommlet F, Schweiger $\mathrm{T}$, et al. Intraoperative extracorporeal membrane oxygenation and the possibility of postoperative prolongation improve survival in bilateral lung transplantation. J Thorac Cardiovasc Surg. 2018;155:2193-206.e3. 